\title{
Unconventional Spin Relaxation Involving Localized Vibrational Modes in Ho Single-Atom Magnets
}

\author{
F. Donati® ${ }^{1,2,3}$ S. Rusponi ${ }^{2}{ }^{2}$ S. Stepanow, ${ }^{4}$ L. Persichetti, ${ }^{4,5}$ A. Singha, ${ }^{1,2,3}$ D. M. Juraschek $\odot,{ }^{4,6}$ \\ C. Wäckerlin $\odot,{ }^{2,7}$ R. Baltic, ${ }^{2}$ M. Pivetta $\odot,{ }^{2}$ K. Diller $\odot,{ }^{2}$ C. Nistor, ${ }^{4}$ J. Dreiser $\odot,{ }^{8}$ K. Kummer, ${ }^{9}$ E. Velez-Fort, ${ }^{9}$ \\ N. A. Spaldin $\odot,{ }^{4}$ H. Brune $\odot{ }^{2}$ and P. Gambardella ${ }^{4}$ \\ ${ }^{1}$ Center for Quantum Nanoscience, Institute for Basic Science (IBS), 03760 Seoul, Republic of Korea \\ ${ }^{2}$ Institute of Physics, École Polytechnique Fédérale de Lausanne (EPFL), Station 3, CH-1015 Lausanne, Switzerland \\ ${ }^{3}$ Department of Physics, Ewha Womans University, Seoul 03760, Republic of Korea \\ ${ }^{4}$ Department of Materials, ETH Zurich, Hönggerbergring 64, CH-8093 Zurich, Switzerland \\ ${ }^{5}$ Department of Sciences, Roma Tre University, I-00146, Roma, Italy \\ ${ }^{6}$ Harvard John A. Paulson School of Engineering and Applied Sciences, Harvard University, Cambridge, Massachusetts 02138, USA \\ ${ }^{7}$ Institute of Physics of the Czech Academy of Sciences, Cukrovarnická 10, 16200 Prague 6, Czech Republic \\ ${ }^{8}$ Swiss Light Source (SLS), Paul Scherrer Institute (PSI), CH-5232 Villigen PSI, Switzerland \\ ${ }^{9}$ European Synchrotron Radiation Facility (ESRF), F-38043 Grenoble, France
}

(Received 24 September 2019; revised manuscript received 20 December 2019; accepted 17 January 2020; published 21 February 2020)

\begin{abstract}
We investigate the spin relaxation of Ho single atom magnets on $\mathrm{MgO} / \mathrm{Ag}(100)$ as a function of temperature and magnetic field. We find that the spin relaxation is thermally activated at low field, while it remains larger than $1000 \mathrm{~s}$ up to $30 \mathrm{~K}$ and $8 \mathrm{~T}$. This behavior contrasts with that of single molecule magnets and bulk paramagnetic impurities, which relax faster at high field. Combining our results with density functional theory, we rationalize this unconventional behavior by showing that local vibrations activate a twophonon Raman process with a relaxation rate that peaks near zero field and is suppressed at high field. Our work shows the importance of these excitations in the relaxation of axially coordinated magnetic atoms.
\end{abstract}

DOI: 10.1103/PhysRevLett.124.077204

The exploration of magnetic atomic-scale units for logical operations [1] and data storage [2] has revived interest in the spin relaxation mechanisms of systems composed of one or few magnetic atoms in a nonmagnetic environment. Early studies on polymetallic $[3,4]$ and single ion molecular magnets (SIMMs) [5-7] showed that the spin relaxation below $4 \mathrm{~K}$ can be sufficiently slow to enable the observation of magnetic hysteresis [8,9]. Effective strategies employed to increase the spin relaxation time, hence the temperature range of magnetic bistability, rely on engineering the ligand field so as to maximize the uniaxial magnetic anisotropy and, consequently, the effective barrier for magnetization reversal $U_{\text {eff }}$ [10-12]. Recently, it has been shown that the hysteretic behavior can be reinforced by minimizing underbarrier relaxation pathways and engineering the coupling between the vibrational modes of the ligands and the magnetic states of the core [13-15]. Combining these approaches has enabled hysteresis up to unprecedented temperatures of 72 [16] and $80 \mathrm{~K}$ [17].

Individual magnetic atoms adsorbed on a single crystal surface allow for exploring magnetic anisotropy [18-21] and bistability [22-24] in a simplified ligand environment. In such a configuration, one can achieve maximal uniaxial anisotropy and minimal coordination of the magnetic atoms $[21,22,25,26]$. As will be shown here, the latter has the additional effect of reducing the coupling of the spin to the phonon modes of the substrate. The validity of this approach was demonstrated by the observation of magnetic remanence up to $40 \mathrm{~K}$ in $\mathrm{Ho}$ atoms on $\mathrm{MgO} / \mathrm{Ag}(100)$, first with $\mathrm{x}$-ray absorption spectroscopy (XAS) and magnetic circular dichroism (XMCD) [22], and subsequently confirmed by scanning tunneling microscopy (STM) $[24,27]$. These single-bond-coordinated atom magnets are ideal systems for investigating the fundamental mechanisms causing magnetization reversal. Addressing these mechanisms requires measuring the lifetime of the magnetic states as a function of temperature and magnetic field. For systems with very long lifetimes, these measurements can be performed with accurate statistics using ensemble-averaging techniques.

Here, we combine XAS and XMCD experiments with density functional theory (DFT) to shed light on the magnetic reversal of $\mathrm{Ho}$ atoms on $\mathrm{MgO} / \mathrm{Ag}(100)$. By recording the time evolution of the XMCD intensity, we directly access the magnetic lifetime $\tau$ as a function of temperature $T$ and external magnetic field $B$. At $B=0.01 \mathrm{~T}$, the Ho atoms show $\tau \approx 1500$ s up to $10 \mathrm{~K}$, and an exponential decrease of $\tau$ with $T$ down to about $100 \mathrm{~s}$ at $30 \mathrm{~K}$. Remarkably, we observe that the lifetime measured at $B=6.8 \mathrm{~T}$ does not show any significant variation with temperature, and remains constant 
(a)

(b)
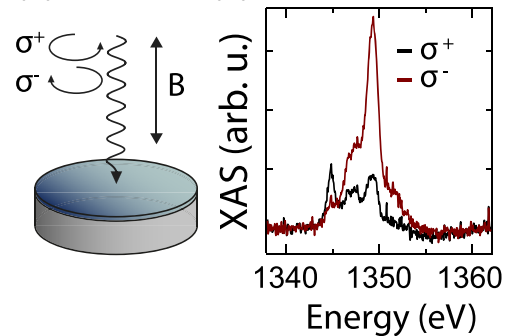

(c)

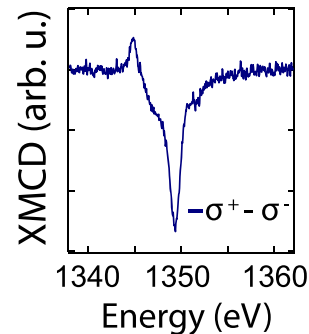

(d)

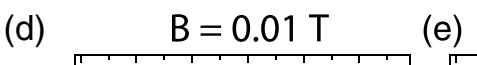

(e) $\quad B=6.8 \mathrm{~T}$
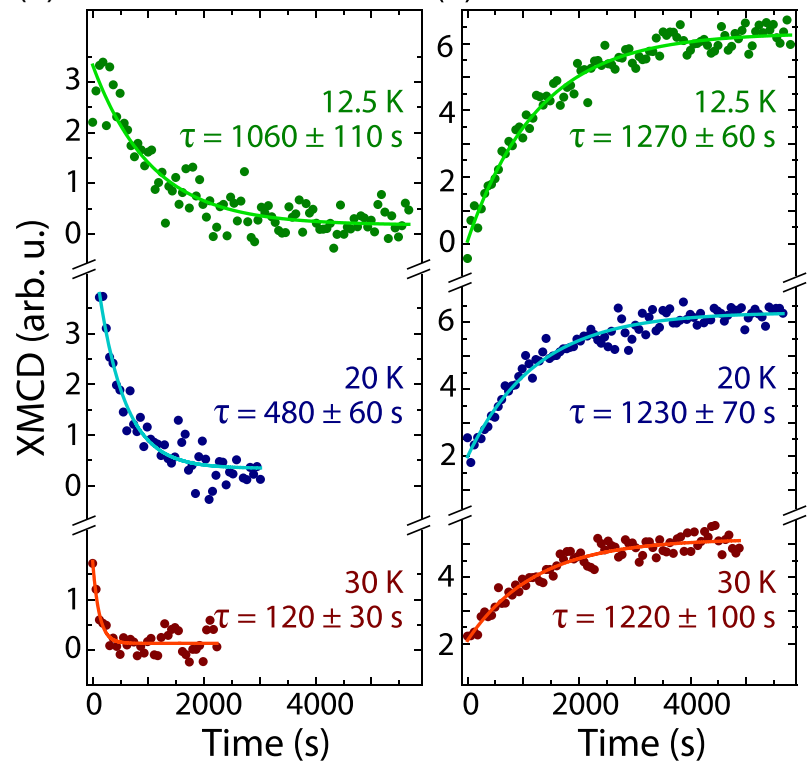

FIG. 1. (a) Sketch of the measurement geometry. (b) XAS and (c) $\mathrm{XMCD}$ of the $\mathrm{Ho}$ atoms on $\mathrm{MgO} / \mathrm{Ag}(100)$ at the $M_{5}$ edge ( $B=6.8 \mathrm{~T}, T=2.5 \mathrm{~K}$, Ho coverage $\Theta_{\mathrm{Ho}} \approx 0.015 \mathrm{ML}$ ). (d) Time evolution of the peak XMCD intensity at $B=0.01 \mathrm{~T}$ after saturating the ensemble at $+6.8 \mathrm{~T}$, and (e) at $B=+6.8 \mathrm{~T}$ after saturating the ensemble at $-6.8 \mathrm{~T}$.

at about $1200 \mathrm{~s}$ up to $30 \mathrm{~K}$. Field-dependent characterization of the magnetic lifetime indicates that suppression of the reversal mechanism occurs above $B=0.3 \mathrm{~T}$. We use DFT to show that only local vibrational modes of the Ho atoms allow for an efficient exchange of energy and momentum between the spin and phonon reservoirs. We propose that this frustrated exchange of energy is responsible for the extremely long magnetic lifetimes of Ho atoms.

The XAS and XMCD measurements were performed at the EPFL/PSI X-Treme beam line of the Swiss Light Source [28] and at the ID32 beam line of the European Synchrotron Radiation Facility [29]. All the measurements were performed in normal incidence geometry with circularly polarized light in total electron yield mode with the external magnetic field parallel to the x-ray beam, see Fig. 1(a) [30]. The magnetic lifetime of the ensemble of Ho atoms was obtained by recording the evolution of the XMCD signal both at low $(B=0.01 \mathrm{~T})$ and high fields (up to $B=8 \mathrm{~T}$ ) after having stabilized the sample temperature

at the desired value. The low field measurements were performed by (i) saturating the ensemble at the largest positive magnetic field, (ii) sweeping the field to $B=$ $+0.01 \mathrm{~T}$ at the maximum rate of $33 \mathrm{mT} / \mathrm{s}$, and (iii) acquiring the XAS signal for the two circular polarizations at the maximum of the $\mathrm{Ho}_{5}$ absorption edge as a function of time [30]. High-field measurements were recorded following a similar procedure after saturating the ensemble at the largest negative field.

Figure 1(b) shows the XAS spectra acquired at the Ho $M_{5}$ edge for the two circular polarizations. The difference gives the XMCD shown in Fig. 1(c). Ho atoms on $\mathrm{MgO} / \mathrm{Ag}(100)$ present a strong out-of-plane magnetic anisotropy, with the ground state being a magnetic doublet [22,24] separated by a few tens of $\mathrm{meV}$ from the first excited states [27]. For such an Ising-like system, the intensity of the XMCD peak reflects the average occupation of the lowest magnetic doublet. Therefore, the time evolution of the peak provides the lifetime of the magnetic states [22,23,31].

Figure 1(d) shows the evolution of the XMCD intensity at low magnetic field and three different temperatures. The data fit well to a single exponential function with vanishing asymptotic magnetization, yielding the characteristic lifetime $\tau$ of the Ho atoms. For increasing temperatures, we observe a decrease of the lifetime from $1060 \pm 110 \mathrm{~s}$ at $12.5 \mathrm{~K}$ to $120 \pm 30 \mathrm{~s}$ at $30 \mathrm{~K}$. For bulk paramagnetic impurities [32,33] and molecular magnets [3-6,10-12,34], the well-known reduction of the magnetic lifetime with increasing temperature is attributed to spinphonon scattering. These mechanisms become increasingly efficient as the number of phonons available for the absorption processes increases with temperature [35]. Figure 1(e) shows the evolution of the XMCD of Ho atoms recorded at $B=6.8 \mathrm{~T}$. Also in this case, we observe an exponential time dependence of the XMCD. The positive asymptotic value reflects the intensity of the dichroism at the maximum available field, which decreases with the sample temperature. However, differently from the low field case, $\tau$ is here unaffected by temperature and remains constant at a value of about $1200 \mathrm{~s}$.

Figure 2 shows $\tau$ as a function of temperature measured at low and high fields. Below $10 \mathrm{~K}$, the relaxation rate is dominated by the scattering with the secondary electrons generated in the $\mathrm{x}$-ray absorption process $[22,31,36]$. These hot electrons represent a temperature-independent extrinsic source of magnetic relaxation that can only be minimized by reducing the photon flux while still having a sufficient $\mathrm{x}$-ray absorption signal. Above $10 \mathrm{~K}, \tau$ behaves very differently depending on the applied field. At $B=0.01 \mathrm{~T}, \tau$ follows an exponential behavior typical of an activated process up to the maximum measured temperature of $30 \mathrm{~K}$. At $B=6.8 \mathrm{~T}, \tau$ remains constant and independent of temperature. This behavior is very different from that of other SIMMs [6,10-12,31,37] and single atom magnets [23], which all show increased spin relaxation at high field. 


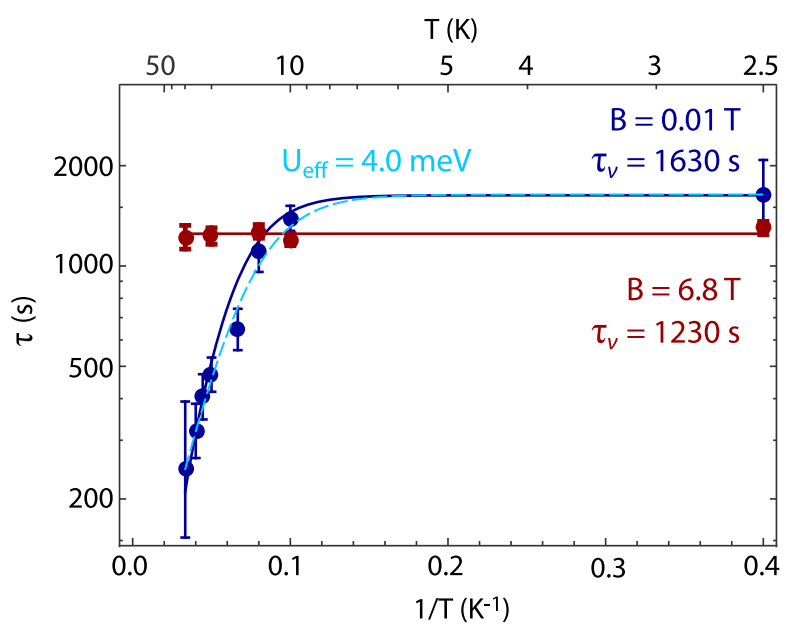

FIG. 2. Magnetic lifetime $\tau$ versus temperature for $B=0.01$ (blue dots) and $B=6.8 \mathrm{~T}$ (red dots). The cyan dashed line is a fit with Eq. (1). Blue and red solid lines are fits to the low and high field data, respectively, using the two-phonon model described in the text.

The temperature dependence of $\tau$ at $B=0.01 \mathrm{~T}$ can be fit by

$$
\tau^{-1}=\tau_{\nu}^{-1}+\left[\tau_{0} \exp \left(U_{\mathrm{eff}} / k_{B} T\right)\right]^{-1},
$$

where $\tau_{\nu}=1630 \mathrm{~s}$ is the photon-limited lifetime due to the secondary electrons [22] and the exponential term describes a thermally activated relaxation with an effective barrier $U_{\text {eff }}=4 \pm 1 \mathrm{meV}$ and a prefactor $\tau_{0}=60 \pm 30 \mathrm{~s}$ [30]. Such an exponential temperature behavior is conventionally associated with a two-phonon Orbach process involving an excited spin state, whose separation from the ground state defines the activation barrier [35]. However, typical prefactors for the Orbach process range from $10^{-6}$ to $10^{-11} \mathrm{~s}[6,8,14,32,38]$, which are orders of magnitude smaller than the $\tau_{0}$ estimated for Ho. Additionally, STM experiments show that the excited states lie at least $15 \mathrm{meV}$ above the ground state doublet [24,27], which is not compatible with the barrier derived from the fit. In fact, even qualitatively, the suppression of spin reversal at high fields is neither compatible with a conventional Orbach mechanism, which predicts an increase of the relaxation rate due to the Zeeman shift of the levels defining the activation barrier, nor with a Raman process activated by delocalized substrate phonons, whose relaxation rate also increases with increasing Zeeman splitting of the lowest doublet [30].

To obtain further insight into the field dependence, we measure the magnetic lifetime of Ho atoms at two selected temperatures, 2.5 and $22 \mathrm{~K}$, as a function of magnetic field. As shown in Fig. 3, at 2.5 K, $\tau$ is constant within the error bars, remaining well above $1000 \mathrm{~s}$ up to $7 \mathrm{~T}$. On the other hand, at $22 \mathrm{~K}, \tau$ decreases strongly below $0.3 \mathrm{~T}$, see inset, whereas no clear trend is visible at higher field [39].

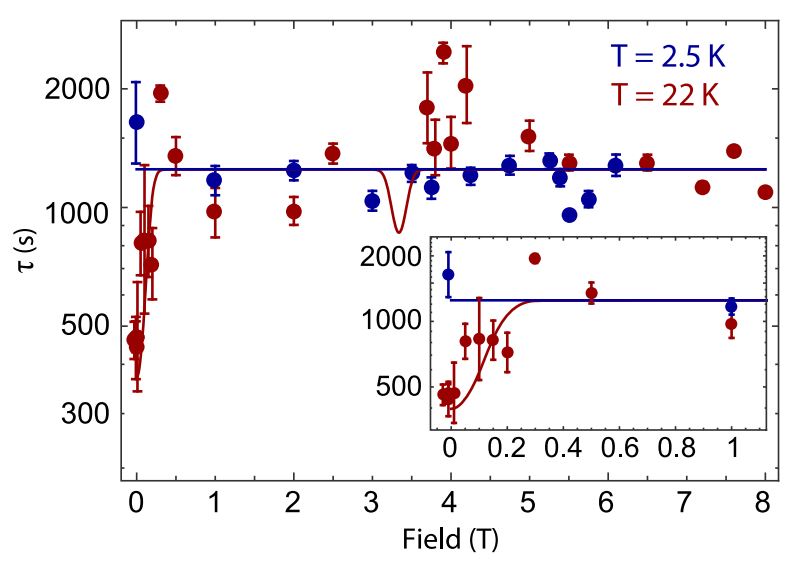

FIG. 3. Magnetic lifetime $\tau$ versus magnetic field measured at 2.5 (blue dots) and $22 \mathrm{~K}$ (red dots). Blue and red solid lines are fits using the two-phonon model described in the text. Inset: magnification of the field range below $B=1 \mathrm{~T}$.

In contrast with this behavior, a faster magnetic relaxation at high field is typically observed in SIMMs, which is ascribed to the direct transition between the two states of the lowest spin doublet mediated by the emission or absorption of a phonon. As the splitting of the magnetic levels increases with the applied field, more phonon states become available for these transitions, hence their relaxation rate increases as well [35]. For Ho single atom magnets, the absence of temperature- and field-dependent relaxation from 0.3 to $8 \mathrm{~T}$ indicates that the direct relaxation process is slower than the photon-induced relaxation up to the longest timescale available in our experiments (thousands of seconds).

The magnetic lifetime of $\mathrm{Ho}$ atoms on $\mathrm{MgO} / \mathrm{Ag}(100)$ is thus not compatible with conventional models of spin relaxation based on either direct, Orbach, or Raman processes, as none of these models can explain an activated process that takes place exclusively at low field. A common feature of these models is that the phonon density of states (DOS) is assumed to be a continuum. This assumption is questionable in the case of adatoms, for which strong resonances are expected in correspondence of the local vibrational modes of the atom-surface complex. DFT calculations of a Ho atom adsorbed on a slab of 3 monolayers (ML) of $\mathrm{MgO}$ [30] provide insight into the vibrational structure of our system. Figure 4(a) shows the calculated phonon DOS projected onto the Ho atom and the $\mathrm{MgO}$ slab, respectively, for a Ho atom adsorbed on top of a surface oxygen ion [22,26]. The $\mathrm{MgO}$ phonon DOS shows a linear behavior owing to the two-dimensional phonon dispersion of the ultrathin slab. The Ho phonon DOS, on the other hand, presents two strong resonances corresponding to a doubly degenerate in-plane Ho-O vibration with an energy at the Brillouin zone center of $E_{\|}=4.7 \mathrm{meV}$ and an out-of-plane Ho-O vibration with an energy of $E_{\perp}=8.6 \mathrm{meV}$. In the $C_{4 v}$ symmetry of the adsorption site, the two in-plane modes are degenerate, see 


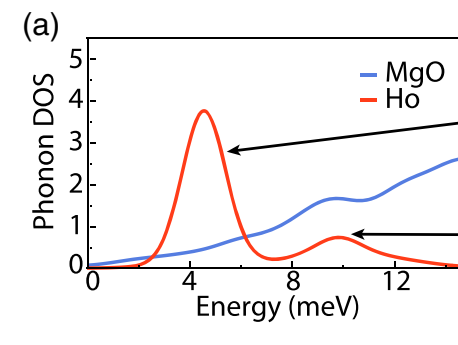

(c)

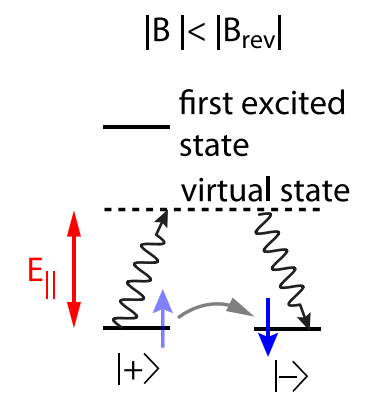

(d) (b)

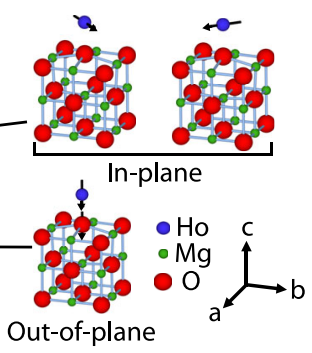

$$
\text { (c) }
$$

FIG. 4. (a) Calculated low-energy phonon DOS projected onto the Ho atom and $\mathrm{MgO}$ slab (see Ref. [30] for the full range plot). (b) Eigenvector of the modes with large displacement of the Ho atoms. (c) Schematics of the two-phonon process responsible for the thermally activated relaxation at low field and high temperature. (d) In high field, this mechanism is suppressed due to the energy mismatch of the lowest states.

Fig. 4(b). Remarkably, the energy of the in-plane modes matches the activation barrier $U_{\text {eff }}$ inferred from our measurements.

Motivated by these observations, we surmise that the peculiar magnetic behavior of Ho single atom magnets is influenced by the contribution of local vibrational modes to the spin-phonon coupling $[15,40]$. The relaxation process is thermally activated and occurs only at low magnetic fields, i.e., when the two spin levels of the lowest doublet are very close in energy. Further, the exceedingly long $\tau_{0}$ suggests that relaxation occurs via a higher order process involving the simultaneous absorption and emission of more than one phonon. Based on these assumptions, we develop a model based on the coupling of a single spin to local vibration modes via a two-phonon Raman process [41]. Since the relaxation due to quantum tunneling of the magnetization and direct processes is slower than that induced by photon absorption, these processes are not included in the model. The spin reversal from $|+\rangle$ to $|-\rangle$ and back occurs at a rate $\tau_{s}(B, T)^{-1}=\Gamma(B, T)\left[1+\exp \left(-\Delta / k_{B} T\right)\right]$, with the transition probability obtained from the twophonon integral $[35,41]$

$\Gamma(B, T)=\kappa \int \rho_{\mathrm{Ho}}(\omega, T) \rho_{\mathrm{Ho}}\left(\omega+\frac{\Delta}{\hbar}, T\right) \exp \frac{\hbar \omega+\Delta}{k_{B} T} d \omega$.

Here, $\omega$ and $\omega+(\Delta / \hbar)$ are the frequencies of the two phonons involved in the transition, $\Delta=2 \mu_{\mathrm{Ho}} B$ is the splitting between the two lowest states with $\mu_{\mathrm{Ho}}=$ $10.1 \mu_{B}$ [24], and $\kappa$ is the scaling factor that is taken as a free parameter of the model [30]. The phonon energy density

$$
\rho_{\text {Но }}(\omega, T)=D_{\text {Но }}(\omega) \hbar \omega\left[\exp \left(\frac{\hbar \omega}{k_{B} T}\right)-1\right]^{-1}
$$

only includes the DOS of the local modes with finite projected displacement of the Ho atoms, $D_{\mathrm{Ho}}(\omega)$ [red line in Fig. 4(a)]. In the model, this term is implemented by summing two Gaussian peaks with energy and relative amplitude as found in DFT [30], while their width $\Delta E$ is left as a free parameter. Finally, we calculate $\tau(B, T)$ by adding the photon-limited lifetime $\tau_{\nu}$ as in Eq. (1).

As seen from the full curves in Figs. 2 and 3, our model fully reproduces the temperature- and field-dependent data with $\kappa=1.7 \times 10^{-17}$ and $\Delta E=0.17 \mathrm{meV}$. Although we cannot exclude the contribution of other relaxation processes in the high field region dominated by photon-induced relaxation, we remark that such an agreement cannot be achieved if the delocalized acoustic phonon modes from the $\mathrm{MgO}$ substrate were to contribute significantly to the spin relaxation [30]. Therefore, only the modes at $E_{\|}=4.7 \mathrm{meV}$ with a large amplitude on the Ho atoms effectively enable the magnetic reversal. The rationale of the reversal mechanism is sketched in Figs. 4(c) and 4(d). For $B<B_{\text {rev }}$, where $B_{\text {rev }}=\Delta E / 2 \mu_{\mathrm{Ho}}$ is the field required to suppress the reversal, the ground state doublet is close to degeneracy and the two magnetic states can be connected by two local vibrations within their energy broadening $\Delta E$ when sufficient thermal energy is provided. Because of the lower activation energy, the relaxation essentially occurs via the in-plane mode. The specific relaxation path involving a low energy local vibrational mode strongly reduces the effective barrier with respect to the zero-field level splitting, i.e., $U_{\text {eff }}=E_{\|}$. A similar barrier reduction was also observed for Fe-based SIMM, but attributed to anharmonic phonons in resonant coupling with excited spin states [13]. Moreover, applying a large magnetic field lifts the degeneracy of the two states such that the same quantum of energy absorbed from one state cannot be reemitted to reach the opposite spin state. In this case, the relaxation process is suppressed. The suppression of this process with external field has, to our knowledge, not been reported so far. The model additionally predicts relaxation by a two phonon process involving an in-plane and an out-of-plane mode, with the difference in energy matching the Zeeman spitting at $3.7 \mathrm{~T}$. The related dip predicted in the curve at high temperature of Fig. 3 is, however, too shallow to be detected with sufficient significance [42].

The linewidth of the spin-flip transition is broadened by the finite lifetime of the vibrational mode [41] as well as by the spread in energy of the Ho nuclear states due to the hyperfine interaction $[33,43,44]$. By modeling the low-field 
and high-temperature data of Fig. 3, we infer that $\Delta E=0.17 \pm 0.07 \mathrm{meV}$. This value essentially coincides within the error bars with the expected hyperfine splitting of Ho atoms $[33,43,45]$, suggesting that this contribution dominates over finite lifetime broadening of the vibrational mode.

In conclusion, single atom magnets at surfaces provide an ideal test for models of spin-lattice relaxation in the presence of strongly localized vibrational modes. Our work shows that, for a single spin system, the presence of local vibrational modes leads to a peak in the Raman relaxation rates near zero field, which is suppressed at high field. For axially coordinated Ho single atom magnets on $\mathrm{MgO}$, we identified the relevant thermal relaxation mechanism as a two-phonon Raman process involving the in-plane displacement of the $\mathrm{Ho}$ atom with respect to the $\mathrm{O}$ adsorption site. Such an unconventional spin relaxation mechanism might be relevant also in single molecule magnets with a phonon density of states dominated by localized vibrational resonances.

We acknowledge funding from the Swiss National Science Foundation (SNSF) through Grants No. 200020_157081/1, No. 200021_146715/1, No. PZ00P2_142474, and No. 200021_163225, from ETH Zürich and from the Institute of Basic Science, Korea through the Project No. IBS319 R027-D1.

[1] A. A. Khajetoorians, J. Wiebe, B. Chilian, and R. Wiesendanger, Science 332, 1062 (2011).

[2] S. Loth, S. Baumann, C. P. Lutz, D. M. Eigler, and A. J. Heinrich, Science 335, 196 (2012).

[3] R. Sessoli, D. Gatteschi, A. Caneschi, and M. A. Novak, Nature (London) 365, 141 (1993).

[4] M. Mannini, F. Pineider, C. Danieli, L. Totti, F. Sorace, P. Sainctavit, M.-A. Arrio, E. Otero, L. Joly, J. C. Cezar, A. Cornia, and R. Sessoli, Nature (London) 468, 417 (2010).

[5] N. Ishikawa, M. Sugita, T. Ishikawa, S.-y. Koshihara, and Y. Kaizu, J. Am. Chem. Soc. 125, 8694 (2003).

[6] R. Westerström, J. Dreiser, C. Piamonteze, M. Muntwiler, S. Weyeneth, H. Brune, S. Rusponi, F. Nolting, A. Popov, S. Yang, L. Dunsch, and T. Greber, J. Am. Chem. Soc. 134, 9840 (2012).

[7] J. M. Zadrozny, D. J. Xia, M. Atanasov, G. J. Long, F. Grandjean, F. Neese, and J. R. Long, Nat. Chem. 5, 577 (2013).

[8] D. Gatteschi, R. Sessoli, and J. Villain, Molecular Nanomagnets (Oxford University Press, Oxford, 2006).

[9] J. Bartolomé, F. Luis, and J. F. Fernández, Molecular Magnets (Springer, Berlin, 2014).

[10] L. Ungur, J. J. Le Roy, I. Korobkov, M. Murugesu, and L. F. Chibotaru, Angew. Chem. Int. Ed. 53, 4413 (2014).

[11] F. Pointillart, K. Bernot, S. Golhen, B. Le Guennic, T. Guizouarn, L. Ouahab, and O. Cador, Angew. Chem. Int. Ed. 54, 1504 (2015).
[12] Y.-C. Chen, J.-L. Liu, L. Ungur, J. Liu, Q.-W. Li, L.-F. Wang, Z.-P. Ni, L. F. Chibotaru, X.-M. Chen, and M.-L. Tong, J. Am. Chem. Soc. 138, 2829 (2016).

[13] A. Lunghi, F. Totti, R. Sessoli, and S. Sanvito, Nat. Commun. 8, 14620 (2017).

[14] C. A. P. Goodwin, F. Ortu, D. Reta, N. F. Chilton, and D. P. Mills, Nature (London) 548, 439 (2017).

[15] L. Escalera-Moreno, J. J. Baldoví, A. Gaita-Arińo, and E. Coronado, Chem. Sci. 9, 3265 (2018).

[16] K. R. McClain, C. A. Gould, K. Chakarawet, S. J. Teat, T. J. Groshens, J. R. Long, and B. G. Harvey, Chem. Sci. 9, 8492 (2018).

[17] F.-S. Guo, B. M. Day, Y.-C. Chen, M.-L. Tong, A. Mansikkamaki, and R.A. Layfield, Science 362, 1400 (2018).

[18] P. Gambardella, S. Rusponi, M. Veronese, S. S. Dhesi, C. Grazioli, A. Dallmeyer, I. Cabria, R. Zeller, P. H. Dederichs, K. Kern, C. Carbone, and H. Brune, Science 300, 1130 (2003).

[19] C. F. Hirjibehedin, C.-Y. Lin, A. F. Otte, M. Ternes, C. P. Lutz, B. A. Jones, and A. J. Heinrich, Science 317, 1199 (2007).

[20] B. Bryant, A. Spinelli, J. J. T. Wagenaar, M. Gerrits, and A. F. Otte, Phys. Rev. Lett. 111, 127203 (2013).

[21] I. G. Rau, S. Baumann, S. Rusponi, F. Donati, S. Stepanow, L. Gragnaniello, J. Dreiser, C. Piamonteze, F. Nolting, S. Gangopadhyay, O. R. Albertini, R. M. Macfarlane, C. P. Lutz, B. A. Jones, P. Gambardella, A. J. Heinrich, and H. Brune, Science 344, 988 (2014).

[22] F. Donati, S. Rusponi, S. Stepanow, C. Wäckerlin, A. Singha, L. Persichetti, R. Baltic, K. Diller, F. Patthey, E. Fernandes, J. Dreiser, Ž. Šljivančanin, K. Kummer, C. Nistor, P. Gambardella, and H. Brune, Science 352, 318 (2016)

[23] R. Baltic, M. Pivetta, F. Donati, C. Wäckerlin, A. Singha, J. Dreiser, S. Rusponi, and H. Brune, Nano Lett. 16, 7610 (2016).

[24] F. D. Natterer, K. Yang, W. Paul, P. Willke, T. Choi, T. Greber, A. J. Heinrich, and C. P. Lutz, Nature (London) 543, 226 (2017).

[25] S. Baumann, F. Donati, S. Stepanow, S. Rusponi, W. Paul, S. Gangopadhyay, I. G. Rau, G. E. Pacchioni, L. Gragnaniello, M. Pivetta, J. Dreiser, C. Piamonteze, C. P. Lutz, R. M. Macfarlane, B. A. Jones, P. Gambardella, A. J. Heinrich, and H. Brune, Phys. Rev. Lett. 115, 237202 (2015).

[26] E. Fernandes, F. Donati, F. Patthey, S. Stavrić, Z. Šljivančanin, and H. Brune, Phys. Rev. B 96, 045419 (2017).

[27] F. D. Natterer, F. Donati, F. Patthey, and H. Brune, Phys. Rev. Lett. 121, 027201 (2018).

[28] C. Piamonteze, U. Flechsig, S. Rusponi, J. Dreiser, J. Heidler, M. Schmidt, R. Wetter, M. Calvi, T. Schmidt, H. Pruchova, J. Krempasky, C. Quitmann, H. Brune, and F. Nolting, J. Synchrotron Radiat. 19, 661 (2012).

[29] K. Kummer, A. Fondacaro, E. Jimenez, E. Vélez-Fort, A. Amorese, M. Aspbury, F. Yakhou-Harris, P. van der Linden, and N. B. Brookes, J. Synchrotron Radiat. 23, 464 (2016).

[30] See Supplemental Material at http://link.aps.org/supplemental/ 10.1103/PhysRevLett.124.077204 for additional information on experimental and computational details, estimation of the 
effective energy barrier, and models for magnetic relaxation processes.

[31] C. Wäckerlin, F. Donati, A. Singha, R. Baltic, S. Rusponi, K. Diller, F. Patthey, M. Pivetta, Y. Lan, S. Klyatskaya, M. Ruben, H. Brune, and J. Dreiser, Adv. Mater. 28, 5195 (2016).

[32] G. H. Larson and C. D. Jeffries, Phys. Rev. 141, 461 (1966).

[33] R. Giraud, W. Wernsdorfer, A. M. Tkachuk, D. Mailly, and B. Barbara, Phys. Rev. Lett. 87, 057203 (2001).

[34] F. Liu, G. Velkos, D. S. Krylov, L. Spree, M. Zalibera, R. Ray, N. A. Samoylova, C.-H. Chen, M. Rosenkranz, S. Schiemenz, F. Ziegs, K. Nenkov, A. Kostanyan, T. Greber, A. U. B. Wolter, M. Richter, B. Büchner, S. M. Avdoshenko, and A. A. Popov, Nat. Commun. 10, 571 (2019).

[35] A. Abragam and B. Bleaney, Electron Paramagnetic Resonance of Transition Ions (Clarendon Press, Oxford, 1970).

[36] J. Dreiser, R. Westerström, C. Piamonteze, F. Nolting, S. Rusponi, H. Brune, S. Yang, A. Popov, L. Dunsch, and T. Greber, Appl. Phys. Lett. 105, 032411 (2014).

[37] M. Studniarek, C. Wäckerlin, A. Singha, R. Baltic, K. Diller, F. Donati, S. Rusponi, H. Brune, Y. Lan, S. Klyatskaya, M. Ruben, A. P. Seitsonen, and J. Dreiser, Adv. Sci. 6, 1901736 (2019).
[38] N. Ishikawa, M. Sugita, T. Ishikawa, S. Koshihara, and Y. Kaizu, J. Phys. Chem. B 108, 11265 (2004).

[39] We attribute the scattering of the data at high field to possible variations of the photon flux conditions occurring over the different experimental sessions required for the data collection.

[40] S. Roychoudhury and S. Sanvito, Phys. Rev. B 98, 125204 (2018).

[41] D. L. Mills, Phys. Rev. 146, 336 (1966).

[42] A more accurate computation of the relaxation rate for this process would require considering the energy and momentum dependence of the prefactor $\kappa$, which is beyond the scope of the present work.

[43] N. Ishikawa, M. Sugita, and W. Wernsdorfer, J. Am. Chem. Soc. 127, 3650 (2005).

[44] P. R. Forrester, F. Patthey, E. Fernandes, D. P. Sblendorio, H. Brune, and F. D. Natterer, Phys. Rev. B 100, 180405(R) (2019).

[45] Using typical values of $A_{J} \approx 3.5 \mu \mathrm{eV}[33,38]$, we estimate a hyperfine splitting $\Delta E=2 A_{J} \times J_{z} \times I \approx 170$ and $195 \mu \mathrm{eV}$ for two possible multiplet scenarios with ground state $J_{z}=7$ and 8, respectively [24]. 